\title{
STUDY AND DEVELOPMENT OF A DEVICE FOR ACQUISITION OF ECG SIGNALS FOR INSERTION IN A TECHNOLOGICAL ULTRASOUND PLATFORM
}

\section{Matheus A. A. Nogueira*, Eduardo T. Costa.}

\begin{abstract}
The Brazilian Government is financing the development of an ultrasound platform to dominate and transfer medical ultrasound technology to the Brazilian industry. All ultrasound imaging equipment is equiped with an ECG module that is the main purpose of this project. Our ECG module contains 2 main circuits: 1) an analogic circuit with instrumentation amplifier and filters to amplify the ECG signal (D1) powered by bateries to isolate patient from the mains; 2) the analog to digital conversion and storage circuitry to allow the main ultrasound system to get and show the ECG signal along with ultrasound images.
\end{abstract}

\section{Key words:}

Ultrasound, ECG, Signal Acquisition.

\section{Introduction}

Aiming at improving the national scenario of health care and medical equipment development in Brazil, UNICAMP is leading six research groups to develop a genuinely Brazilian ultrasound platform that will be equipped with an ECG (ElectroCardioGram) signal module. The present project shows this module developed with circuitry to acquire and amplify ECG signals (Derivation I), using active filters. For patient safety, the analogic circuits are isolated from the mains and linked to the digital circuits via an optocoupler. We have used a microcontroller (PIC32MX220F032B) for the analog-to-digital conversion, storage of the ECG digitized signal in a rotating memory buffer and, at the request of the main software, the acquired data is sent to the ultrasound platform by serial protocol (USB port).

\section{Results and Discussion}

The block diagram shows the circuitry described ahead.

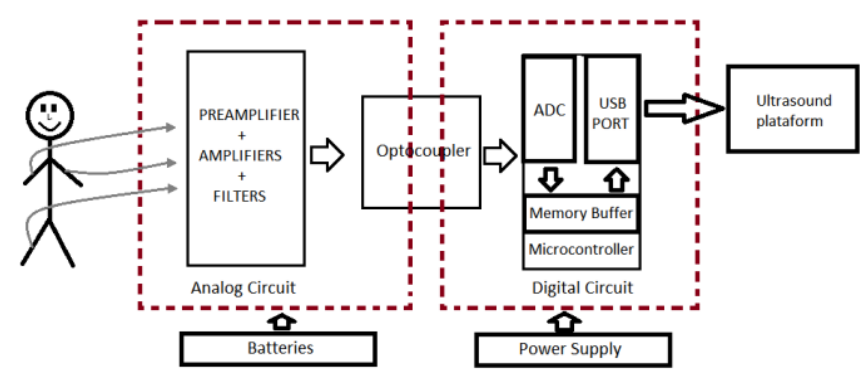

The ECG signal is acquired using Derivation I (electrodes in both arms and right leg) using an instrumentation amplifier (INA122) as preamplifier $(G=65)$. The preamplified signal passes through a sequence of active filters for noise treatment and final amplification (High Pass $-\mathrm{fc}=$ $0.5 \mathrm{~Hz}, \mathrm{G}=3.5$; Low Pass $-\mathrm{fc}=100 \mathrm{~Hz}, \mathrm{G}=3.3$; and Notch $-\mathrm{fn}=60 \mathrm{~Hz}, \mathrm{G}=6)$.

The filtered signal feeds an optocoupler (4N25) to isolate the patient (analog circuit) from the main power supply that feeds the digital circuitry. The analog circuit is powered by $9 \mathrm{~V}$ batteries and, using two voltage regulators (LM7805 and LM7905), creates a symmetrical 5V supply with a circuit to alarm when the batteries are discharged.
The signal, after the optocoupler, is presented to the 10 bits $A / D$ converter that is part of the microcontroller (PIC32MX220F032B). The A/D Reference Voltage consists of a $3 \mathrm{~V}$ precision voltage regulator (REF3030). A 3.3V voltage regulator feeds the microcontroller. The digitized ECG signal is stored in the internal PIC memory (2000 points acquired at $1 \mathrm{kHz}$ sampling frequency). At the request of the main software running in the ultrasound platform, the stored signal is sent via an USB port.

Figure 1 shows the preamplifier output (green) and the $A / D$ input (yellow) of an ECG signal. Figure 2 shows the ECG signal treated and stored in memory.

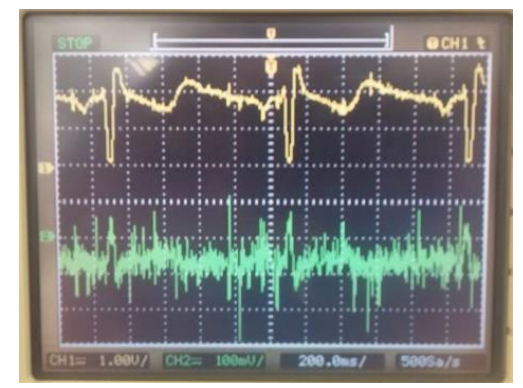

Figure 1

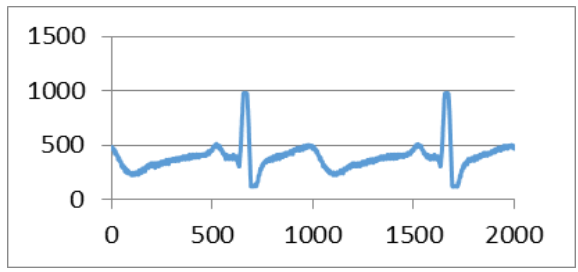

Figure 2

\section{Conclusions}

The ECG acquisition module has been designed, constructed and tested, presenting very good results and is ready to be inserted in our ultrasound platform.

\section{Acknowledgement}

We are grateful to CNPq for the scholarship within the PIBIC Program. We also acknowledge the APD/CEB for technical support. 